“ (C) 2017 IEEE. Personal use of this material is permitted. Permission from IEEE must be obtained for all other uses, in any current or future media, including

reprinting/republishing this material for advertising or promotional purposes, creating new collective works, for resale or redistribution to servers or lists, or reuse of any copyrighted component of this work in other works." 


\title{
Robust Control of a Brachiating Robot
}

\author{
Kim-Doang Nguyen and Dikai Liu
}

\begin{abstract}
This paper investigates the robust control of an underactuated brachiating robot. The control schemes are motivated by the applications that require robots to move through lattice structures, such as the inspection and maintenance of power transmission lines and towers. Inspired by the pendulum-like movements in gibbons' arboreal locomotion, the controllers are designed to synchronize the brachiator with a virtual oscillator. Two schemes are proposed: a modeldependent feedback linearization scheme and a sliding-mode scheme that is independent of the system model. The numerical results illustrate that the proposed schemes are robust to the arbitrary initial configurations of the brachiator and the limitation in the motor torque at the elbow joint. They are able to perform successful fast swing-up and dynamic brachiating in a unified control framework. Furthermore, both controllers enable the underactuated robot to brachiate along a structural member with an upward slope.
\end{abstract}

\section{INTRODUCTION}

Periodic inspection and maintenance are critical to ensuring the healthy condition of power transmission towers as well as the stable operation of the transmission lines they support. Because of the high expenses and many safety concerns involved, this is an emerging problem that robotic systems may offer appealing solutions. Such a system will eliminate the need for the dangerous climbing and operations near high-voltage cables that workers have to carry out in the current practice. The main difficulty is that the complexity of the tower structure poses a tremendous challenge for robotic systems to perform locomotion and to navigate in the complicated workspace away from the ground.

One approach to addressing the locomotion problem is to study animals in order to translate their successful principles to robot and control design. The Hominoidea superfamily, including humans, bonobos, chimpanzees, gorillas, orangutans, and gibbons, are well known for their exceptional climbing skills. Among these, gibbons are the most arboreal and the ones capable of true brachiation [1]. Beside their centralized mass distribution and excellent power-to-weight ratio, gibbons' ability to generate pendulum-like brachiating movements has been studied in [2]-[4].

These studies on gibbons' arboreal locomotion have inspired researchers to investigate the dynamics, to develop control frameworks, and to build robots that can perform brachiation. Brachiating motions may be achieved via simple point-mass models [5], two-link models [2], or five-link models [6], all with theoretically no mechanical energy cost. Recent work in [7] proposes a hybrid mechanical model for

Kim-Doang Nguyen is with the Mechanical Engineering Department at South Dakota State University, Doang. Nguyenesdstate. edu

Dikai Liu is with the Centre for Autonomous Systems at University of Technology Sydney, Australia, Dikai. Liu@uts. edu. au a two-link brachiating robot. Passive-dynamics brachiation is achieved by applying the stable-gait generation strategy developed in [6]. This work is then extended in [8] and [9] as a unified framework for the passive brachiating and walking of a two-link model.

Current research into the passive dynamics of brachiating models has deep implications of how dynamic brachiation is achieved with zero mechanical energy and provides insight into the principles that make gibbons so efficient in moving through arboreal environments. However, passive brachiators have limited practical use as compared to robots that brachiate via active control. Work in [10] and [11] proposes a controller that decomposes complicated brachiating motion into behavior primitives. The behavior-based approach can perform simple behaviors that are pre-composed experimentally. In [12], an improved energy-based control framework for multiple smooth brachiating swings was developed. The framework was further extended to enable the robot to perform both walking and brachiating as well as the transition between them in [13].

The intriguing concept of target dynamics was introduced to encode pendulum-like brachiating motion in [14]-[16]. The core idea of this controller is to directly cancel the system nonlinearity via a Lie-derivative based linearization scheme to turn the brachiator's dynamics into the target dynamics. The target dynamics are then designed to achieve either swing-up or brachiating movements. The target-dynamics controller enables a two-link brachiator to achieve precise brachiating strides by waiting for all kinetic energy to die off after each swing. As a result, each brachiating swing is a replication of the first one. However, a key property of the target dynamics scheme is that the target dynamics are reverse-time symmetric. This means that the brachiator performs exactly mirrored trajectory and reaches a point which is perfectly opposite to the starting point relative to the handhold. Hence, this controller is highly dependent on the initial conditions. In addition, it is intolerant of any limitation in the elbow joint's motor torque.

In this paper, motivated by the limitations of the existing work, we develop two brachiating controllers: a feedbacklinearization scheme and a mode-independent sliding-mode scheme, which are both

- Robust to the arbitrary initial configurations of the robotic brachiator,

- Tolerant of the limitation in the elbow torque,

- Capable of performing fast swing up in the presence of the torque limitation,

- Able to brachiate along a structural member with an upward slope. 


\section{DYNAMIC BRACHIATING}

Consider the model of a two-link brachiating robot. The robot has two passive wrist joints and an active elbow joint (See Fig. 1). This is the same underactuated robot model studied in most robotic brachiating literature, for example, [6], [7], [15]. Here, the properties of the two links are different. There are two active grippers at two ends of the robot which perform necessary grasping for brachiating.

This paper investigates two classes of motion in dynamic brachiating: swing-up motion and brachiating along a structural member. The swing-up motion is defined as the motion of the robot from a straight-down rest configuration to a point on a structural member, i.e. the first grasp. Brachiating along a structural member refers to the swing of the robot from a point on a structural member to the next point on the member.

The brachiator's equation of motion has the following standard form of an underactuated Lagrangian system:

$$
M(q) \ddot{q}+N(\dot{q}, q)=\left[\begin{array}{l}
0 \\
\tau
\end{array}\right]
$$

where $q=\left[\theta_{1}, \theta_{2}\right]^{\top}, M(q)$ is the inertial matrix, and $N(\dot{q}, q)$ represents the nonlinearity, gravity, friction, and the effect of disturbances. The control objectives are to design the input signal to the elbow joint's motor so that the robot can perform the two types of motion described at the beginning of this section. The objectives should be achieved with robustness to initial configurations, motor torque limits, disturbances, and model uncertainties. These objectives must be achieved with one unified control structure, as opposed to different control structures required for different types of motion.
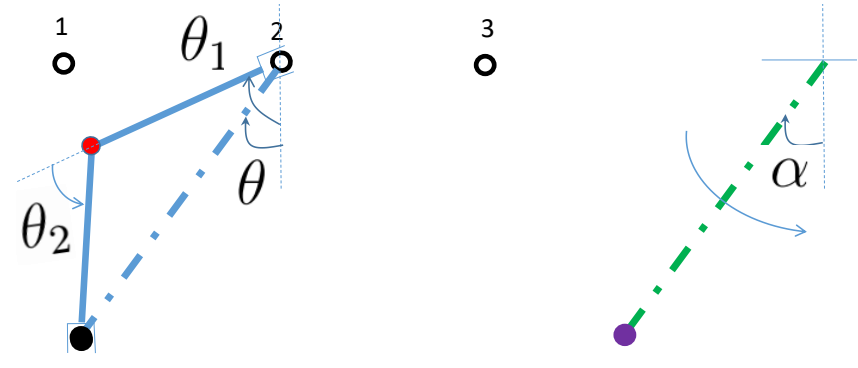

Brachiator

Virtual oscillator

Fig. 1. The control objective is to synchronize the two oscillators $\theta(t)$ and $\alpha(t)$.

\section{A FEEDBACK Linearization Scheme}

The research in this paper is along the line of work on the active control of brachiators in [14]-[16]. The core idea in these papers is to make the brachiator's dynamics identical to the target dynamics, which admit the initial condition of the brachiator. The key property is that the target dynamics are reverse-time symmetric. This means that the brachiator performs exactly mirrored trajectory and reaches a point which is perfectly opposite to the starting point relative to the handhold. In other words, if the brachiator's end-effector starts at a point slightly below the structural member, it would not reach the structural member on the other side of the base. Hence, this controller is highly dependent on the initial conditions. Motivated by this limitation of the targetdynamics based controller, we approach the problem with the idea of oscillator synchronization.

Consider the following oscillator with its own initial conditions

$$
\ddot{\alpha}(t)=-\omega^{2} \alpha(t), \alpha(0)=\alpha_{0}, \dot{\alpha}(0)=\nu_{0} .
$$

This oscillator is reset to the initial state after every swing, i.e. after every time the system state is in the jump set. Define the angle

$$
\theta \triangleq \theta_{1}+0.5 \theta_{2}
$$

which indicates the polar angle of the end-effector.

The key method in this paper is to design a controller that synchronizes the two oscillators $\theta(t)$ and $\alpha(t)$ in Figure 1 . This is fundamentally different from the idea of target dynamics presented in [15]. There, the controller turns the brachiator's dynamics into the target dynamics, which admit the initial conditions of the brachiator. In addition, because of the neutral orbits and the reverse-time symmetry of the target dynamics controller in [15], if the end-effector of the brachiator starts slightly below the structural member, it will never reach the structural member on the other side. It will be clear that the controllers proposed in this paper is robust to arbitrary initial conditions and hence addresses this limitation of the controller in [15].

In order to achieve the synchronization of $\theta(t)$ and $\alpha(t)$, we construct the following auxiliary variable:

$$
x=\dot{\theta}-\dot{\alpha}+\lambda_{1}(\theta-\alpha)
$$

For simplicity, we first consider the case where the system model is known and apply the technique of feedback linearization, proposed in [17] for the swing-up of inverted pendulums, to deal with the nonlinearity in the system.

Taking time derivative of (4) and then substituting (1) yields

$$
\begin{aligned}
\dot{x}= & -\bar{m}_{11} n_{1}-0.5 \bar{m}_{21} n_{1}+\left(\bar{m}_{12}+0.5 \bar{m}_{22}\right)\left(-n_{2}+\tau\right) \\
& -\ddot{\alpha}+\lambda_{1}(\dot{\theta}-\dot{\alpha})
\end{aligned}
$$

where $\bar{m}_{11}, \bar{m}_{12}$, and $\bar{m}_{22}$ are the elements of $M^{-1}(q), n_{1}$ and $n_{2}$ are the elements of $N(\dot{q}, q)$. Here, we use the same assumption in [15] that the brachiating robot is designed such that $\bar{m}_{12}+0.5 \bar{m}_{22}>0$. It is now clear that if we design the control input

$$
\tau=n_{2}+\frac{\bar{m}_{11} n_{1}+0.5 \bar{m}_{21} n_{1}+\ddot{\alpha}-\lambda_{1}(\dot{\theta}-\dot{\alpha})-\lambda_{2} x}{\bar{m}_{12}+0.5 \bar{m}_{22}},
$$

where $\lambda_{2}$ is a positive gain, then (5) becomes

$$
\dot{x}=-\lambda_{2} x .
$$


Therefore, with the control signal in $(6), x(t)$ converges to zero exponentially fast and the convergence rate is determined by $\lambda_{2}$. Consequently, because of (4), $\theta(t)$ converges to $\alpha(t)$ exponentially fast.

The remaining task is to design the initial conditions of $\alpha(t)$ to achieve brachiating tasks. For example, for brachiating along a horizontal structural member, we set $\alpha(0)=$ $-\pi / 2$ and $\dot{\alpha}(0)=0$ so that the virtual pendulum swings from one side of the structural member to the opposite side. Since $\theta(t) \rightarrow \alpha(t)$, the end-effector also swings from one point on the structural member to an opposite point on the member. The brachiating control objective is therefore achieved. For a swing-up task, the initial conditions of $\alpha(t)$ remain unchanged. The brachiator starts from a straightdown rest configuration. Just by synchronizing with the oscillator $\alpha(t)$, the brachiator quickly reaches the structural member. Practical consideration on how to design the oscillator $\alpha$ to achieve certain tasks will be demonstrated in Section V

Remark 1: A special property of the formulation in (4) is that when $x(t) \rightarrow 0$, the variable $\theta(t)$ will slide toward a given trajectory $\alpha(t)$ exponentially fast no matter how $\theta(t)$ is initialized. This property enables the robustness to initial conditions of this controller.

Remark 2: The roles of the links and joints are swapped after every swing, i.e. every time the system state is in the jump set. In particular, after the end-effector reaches the structural member, it becomes the gripping end, or the base. The gripper that is holding on to the ceiling during the last swing now becomes the end-effector. The governing equation of motion (1) hence flips to another form accordingly. Because of this swapping in the vector fields and the resetting of the virtual oscillator $\alpha(t)$, the controller in (6) is an intrinsically nonsmooth controller. Nonetheless, there is no concern about the instability induced by nonsmoothness because the switching is slow and happens only once per swing. During a swing, the controller is smooth.

\section{A Model-Free Sliding-Mode Control Scheme}

In Section III, we present a feedback linearization scheme formulated under the assumption that the system model is precisely known. Existing work on dynamic brachiation also relies on this assumption. As one of the key contributions of this paper, in this section, we develop a brachiating controller that eliminates the need for the knowledge of the system model. The idea originates from the fundamentals of the sliding-mode control framework.

The key principle of the sliding mode control approach is to steer the states toward a vicinity of a certain switching surface [18], in which, the closed-loop response is robust to uncertainties and disturbances [19]. Here, as discussed in the last section, as long as the auxiliary variable $x$ in (4) is driven to and constrained within a small neighborhood of zero, we can design the virtual oscillator to achieve the brachiating tasks. Hence, in the sliding mode framework, we consider $x$ in (4) the switching manifold.
The equation governing the dynamics of $x$ in (5) can be rewritten as follows

$$
\dot{x}=f(\dot{q}, q)+g(q) \tau
$$

where $q=\left[\theta_{1}, \theta_{2}\right]^{\top}, f(\dot{q}, q) \triangleq-\bar{m}_{11} n_{1}-0.5 \bar{m}_{21} n_{1}+$ $\left(\bar{m}_{12}-0.5 \bar{m}_{22}\right) n_{2}-\ddot{\alpha}+\lambda_{1}(\dot{\theta}-\dot{\alpha})$ and $g(q) \triangleq\left(\bar{m}_{12}+\right.$ $\left.0.5 \bar{m}_{22}\right)$. Note that

$$
g(q)=\frac{m_{1} l_{c 1}^{2}+m_{2}\left(l_{1}^{2}-l_{c 2}^{2}\right)+I_{1}-I_{2}}{\operatorname{det}(M)}
$$

where $m_{1}, m_{2}, I_{1}, I_{2}, l_{1}, l_{2}, l_{c 1}$, and $l_{c 1}$ are the masses, moments of inertia, lengths, and location of the centers of mass of the brachiator's two links, respectively. It is obvious that one can always design the brachiator such that $g(q)>0$. For example, a robot with similar link moments of inertia, the centers of mass near the end of the links, and significantly large links' length or mass satisfies this condition.

We are going to design a sliding-mode controller that drive $x(t)$ to a neighborhood of 0 via the following Lyapunov function candidate:

$$
V=0.5 x^{2} \Rightarrow \dot{V}=x \dot{x}
$$

Substituting $\dot{x}$ from $(8)$ in 10 yields

$$
\dot{V}=x g(q)\left(\frac{f(\dot{q}, q)}{g(q)}+\tau\right)
$$

We now establish a bounding function for $f(\dot{q}, q) / g(q)$ in order to derive the form for $\tau$. It is clear that the denominator of $m_{11}, m_{12}$, and $m_{22}$ is $\operatorname{det}(M)$ finite and positive. Their numerators are linear expressions of sine and cosine functions of $\theta_{1}$ and $\theta_{2}$. Hence, $m_{11}, m_{12}$, and $m_{22}$ are bounded for all $\theta_{1}$ and $\theta_{2}$. In addition, $n_{1}$ and $n_{2}$ are quadratic functions of $\dot{q}$ with bounded coefficients, which are sine and cosines functions of $\theta_{1}$ and $\theta_{2}$. Thus, since $\alpha$ and its time derivatives are bounded for all time, we conclude that

$$
\left|\frac{f(\dot{q}, q)}{g(q)}\right|<c_{1}+c_{2}\|\dot{q}\|^{2}
$$

for some known positive $c_{1}$ and $c_{2}$ large enough.

Based on the analysis above, we design

$$
\tau=-\left(\epsilon_{0}+c_{1}+c_{2}\|\dot{q}\|^{2}\right) \operatorname{sat}\left(\frac{x}{\epsilon}\right)
$$

where $\epsilon_{0}>0, \epsilon$ is a boundary layer inside which a continuous control law is imposed, and the saturation function is defined as

$$
\operatorname{sat}(y)= \begin{cases}y, & |y| \leq 1 \\ \operatorname{sgn}(y), & |y|>1\end{cases}
$$

The reason for the boundary layer and the use of the saturation function is to mitigate chattering, which is one of the drawbacks of sliding mode control.

It follows from (11) and (13) that when $|x|>\epsilon$

$$
\begin{aligned}
\dot{V} & =\operatorname{sgn}(x)|x| g(q)\left(\frac{f(\dot{q}, q)}{g(q)}+\left(\epsilon_{0}+c_{1}+c_{2}\|\dot{q}\|^{2}\right) \operatorname{sgn}\left(\frac{x}{\epsilon}\right)\right) \\
& =|x| g(q)\left(\operatorname{sgn}(x) \frac{f(\dot{q}, q)}{g(q)}-\left(\epsilon_{0}+c_{1}+c_{2}\|\dot{q}\|^{2}\right)\right) \\
& \leq-|x| g(q) \epsilon_{0}<0 .
\end{aligned}
$$


Thus, when $x(t)$ is outside the boundary layer indicated by $\epsilon$, the controller steers it toward the inside of the layer as implied by the negative Lyapunov function. Once, $x(t)$ is inside the layer, it is driven by a continuous feedback control scheme.

Similar to the feedback linearization scheme presented in Section III. the remaining task is to design the virtual oscillator such that brachiating task is achieved. In the next section, we implement the proposed two schemes for the swing-up and brachiating tasks.

\section{Simulation Results}

For the purpose of comparison, we employ the identical model of the robotic brachiator implemented in [15]. In particular, one of the links' mass, moment of inertia, length, center of gravity are $3.499 \mathrm{~kg}, 0.090 \mathrm{kgm}^{2}, 0.50 \mathrm{~m}, 0.414 \mathrm{~m}$, respectively, while the other link's mass, moment of inertia, length, center of gravity are $1.232 \mathrm{~kg}, 0.033 \mathrm{kgm}^{2}, 0.5 \mathrm{~m}$, $0.333 \mathrm{~m}$, respectively. The wrist and elbow joints' viscous friction and Coulomb friction coefficients are $0.02 \mathrm{Nm} / \mathrm{s}$, $0.02 \mathrm{Nm}, 0.14 \mathrm{Nm} / \mathrm{s}$, and $0.45 \mathrm{Nm}$, respectively.

\section{A. Fast Swing-up Task}

In the swing-up task, the brachiator needs to swing from a straight-down configuration to a first grasping point on a structural member, which is $0.6 \mathrm{~m}$ from the current grasping point. In the Numerical experiment 1 , the feedback linearization controller, proposed in Section III is implemented with $\omega=3 \mathrm{~Hz}, \alpha(0)=\pi / 2$ and $\dot{\alpha}(0)=0$, and the gains $\lambda_{1}=5$ and $\lambda_{2}=5$. Here, the values of $\lambda_{1}$ and $\lambda_{2}$ are selected large enough to guarantee the synchronization of $\theta(t)$ and $\alpha(t)$ within a short period of time. It is observed from the simulation results that the time required to complete the swing-up motion is $2.2 \mathrm{~s}$. The maximum torque required is $7.7 \mathrm{Nm}$ and the large torque is required during the first $0.7 \mathrm{~s}$ when the brachiator needs to gain momentum. Further experimenting indicates that the maximum torque needed can be reduced by setting a limit on the elbow motor capability. Figure 2 shows a successful swing-up when motor torque is saturated at $\pm 4 \mathrm{Nm}$ indicated in the bottom panel of Fig. 2. We can see that, despite the limited joint torque, the controlled brachiator can still complete the swing-up motion within $2.2 \mathrm{~s}$. This, therefore, illustrates the proposed controller's robustness to the torque capability limitation of the elbow joint's motor. Indeed, the swing-up time may be further reduced by changing the initial conditions of the virtual oscillator with which the brachiator is controlled to synchronize. For example, when the virtual oscillator's initial conditions are changed to $\alpha(0)=0 \mathrm{rad}$ and $\dot{\alpha}(0)=$ $-4.7 \mathrm{rad} / \mathrm{s}$, the swing-up time is reduced to $1.6 \mathrm{~s}$.

In the Numerical experiment 3, the proposed sliding-mode controller (Section IV) is implemented for the swing-up task with $\lambda_{1}=5, \epsilon_{0}+c_{1}=7$, and $c_{2}=0.1$. The virtual oscillator's initial conditions are $\alpha(0)=0$ rad and $\dot{\alpha}(0)=$ $-4.7 \mathrm{rad} / \mathrm{s}$. Fig. 3 shows that the brachiator successfully completes the swing up motion within $1.6 \mathrm{~s}$. Note that this is achieved with limited elbow joint torque and without any knowledge about the system model.
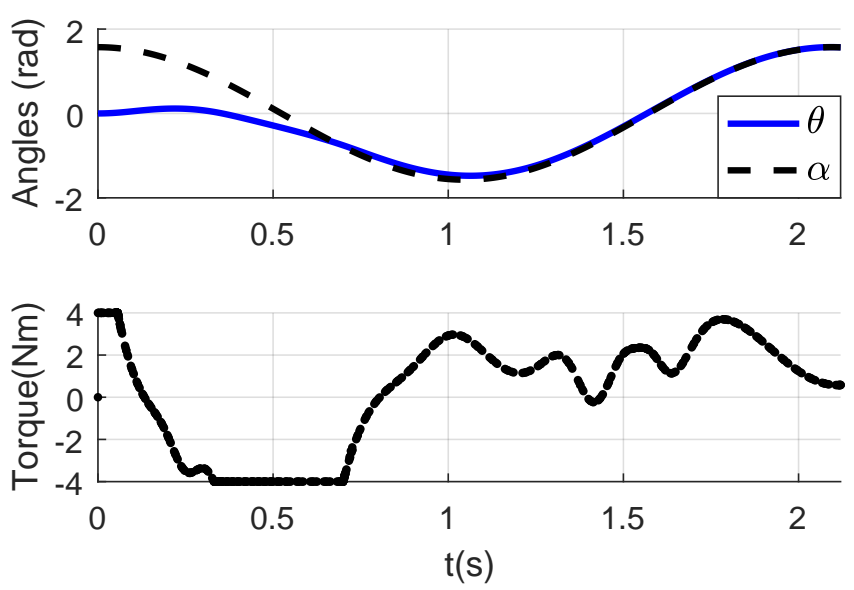

Fig. 2. Numerical experiment 2: Simulation results of a fast swing-up task achieved with the proposed feedback linearization scheme with the motor torque limited in $[-4,4] \mathrm{Nm}$. The swing-up time is approximately $2.2 \mathrm{~s}$.
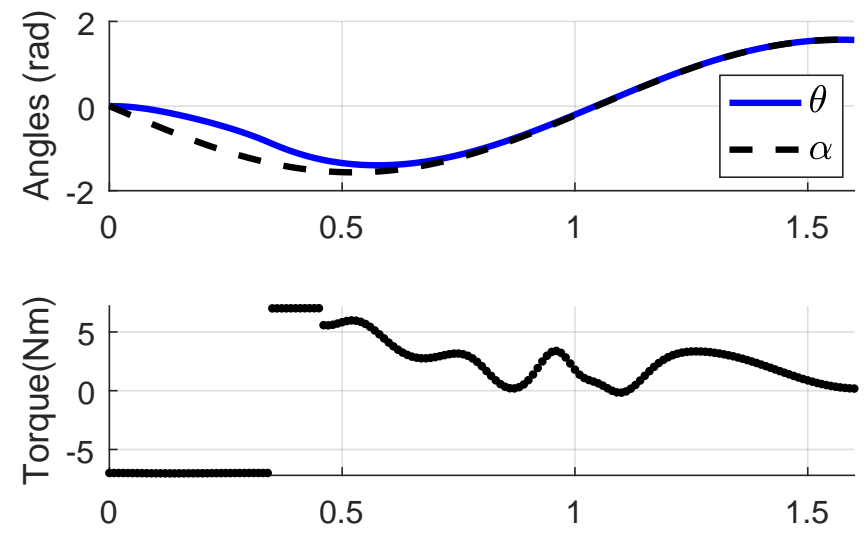

$t(s)$

Fig. 3. Numerical experiment 3: Simulation results of a fast swing-up task achieved with the proposed sliding-mode scheme. The swing-up time is approximately $1.6 \mathrm{~s}$.

\section{B. Brachiating Upwards}

This section demonstrates the capability of the proposed feedback linearization scheme as well as the sliding-mode controller as compared to existing work: brachiating upwards. In this task, the brachiator needs to brachiate along a structural member with an upward slope.

Figure 4 shows the simulation results of six successful brachiating strides along a structural member with a slope of $0.1 \mathrm{rad}$ (5.73 degrees) with the proposed feedbacklinearization scheme. The control parameters are set to the following values: $\lambda_{1}=\lambda_{2}=5$. The brachiator is initialized from the straight-down rest configuration. The virtual oscillator $\alpha(t)$ is initialized and reset after each brachiating stride with $\alpha(0)=-1.8 \mathrm{rad}$ and $\dot{\alpha}(0)=1.3 \mathrm{rad} / \mathrm{s}$. The synchronization of the two oscillators can be observed in Fig. 4 s top panel. The elbow joint's motor torque is limited 
in $[-5,5] \mathrm{Nm}$ as appeared in the bottom panel of Fig. 4 Figure 6 depicts configurations of the brachiator during these six brachiating swings.

Figure 5 demonstrates the performance of the proposed sliding-mode controller during eight successful brachiating strides. The controller is implemented with $\lambda_{1}=5, \epsilon_{0}+c_{1}=$ $3, c_{2}=0.2$, and the boundary layer $\epsilon=0.001$. The brachiator is initialized with $\theta_{1}(0)=-0.6 \mathrm{rad}, \theta_{1}(0)=$ $-1.2 \mathrm{rad}$. The virtual oscillator $\alpha(t)$ is initialized and reset after each brachiating stride with $\alpha(0)=-1.7 \mathrm{rad}$ and $\dot{\alpha}(0)=1.3 \mathrm{rad} / \mathrm{s}$. The top panel of Fig. 5 illustrates the synchronization of the two oscillators. The elbow joint's motor torque time history is provided by the bottom panel of Fig. 5 Figure 7 shows the configurations of the brachiator during these six brachiating swings. One may observe that there are more brachiating strides in Figure 6 than in Fig. 7 . The reason for this is the stride length in the experiment with the sliding mode-controller is smaller than that in the experiment with the feedback linearization scheme. In fact, with both controllers developed in this paper, it is possible to adjust the stride length by changing the initial velocity of the virtual oscillator $\alpha(t)$ after each swing. This will be demonstrated in a future publication.

\section{CONCLUSIONS}

This paper proposes a feedback linearization scheme and a sliding mode controller for a two-link robotic brachiator. The controllers are robust to arbitrary initial conditions of the brachiator and tolerant of the capability limit in the elbow motor torque. The sliding-mode controller removes the dependence on the system modeling that the existing schemes have. Furthermore, both controllers can perform dynamic brachiating along a structural member with an upward slope.
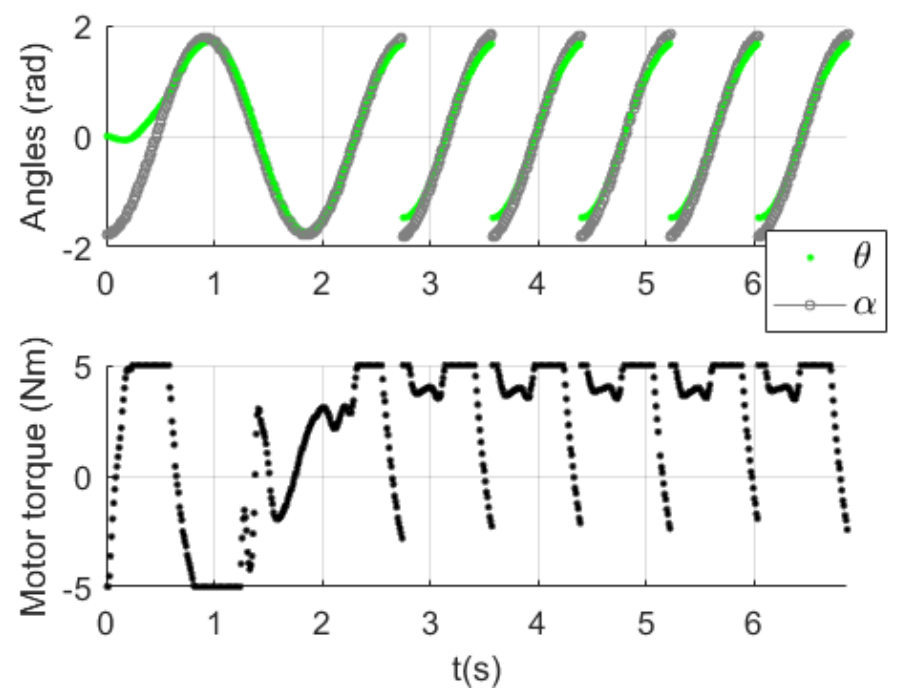

Fig. 4. Numerical experiment 6: The top panel shows the synchronization of $\theta(t)$ and $\alpha(t)$ in six successful upward brachiating strides with the feedback linearization scheme. The bottom panel shows the elbow joint's motor torque with torque capability limited in $[-5,5] \mathrm{Nm}$.
We note that the proposed controllers are able to brachiate upward for a certain range of slope angle only. When the slope is too large, several swings are required for each brachiating stride. The controllers are unable to perform dynamic brachiation if the slope is further increased beyond a certain threshold. One possible way to approach this problem is to add a tail or a trunk to the two-link model to create an extra channel for energy input into the system. Tails are crucial in maintaining balance in animals as well as robots (see [20] and [21]). Our hypothesis is that a tail may substantially enhance a brachiator's ability to gain momentum while swinging to brachiate upwards with a greater slope. Future work will address this issue.

\section{ACKNOWLEDGMENT}

This work is supported in part by South Dakota State University and by the climbing robot project funded by TEPCO (Japan) and the University of Technology Sydney, Australia. The authors also wish to acknowledge the support of the research team members of this project.

\section{REFERENCES}

[1] S. M. Swartz, J. E. Bertram, and A. A. Biewener, "Telemetered in vivo strain analysis of locomotor mechanics of brachiating gibbons," Nature, vol. 342, no. 6247, pp. 270-272, 1989.

[2] J. R. Usherwood and J. E. Bertram, "Understanding brachiation: insight from a collisional perspective," Journal of Experimental Biology, vol. 206, no. 10, pp. 1631-1642, 2003.

[3] J. E. Bertram, "New perspectives on brachiation mechanics," American Journal of Physical Anthropology, vol. 125, no. S39, pp. 100-117, 2004.

[4] F. Michilsens, K. D'Août, and P. Aerts, "How pendulum-like are siamangs? energy exchange during brachiation," American Journal of Physical Anthropology, vol. 145, no. 4, pp. 581-591, 2011.

[5] J. Bertram, A. Ruina, C. Cannon, Y. H. Chang, and M. J. Coleman, "A point-mass model of gibbon locomotion," Journal of Experimental Biology, vol. 202, no. 19, pp. 2609-2617, 1999.

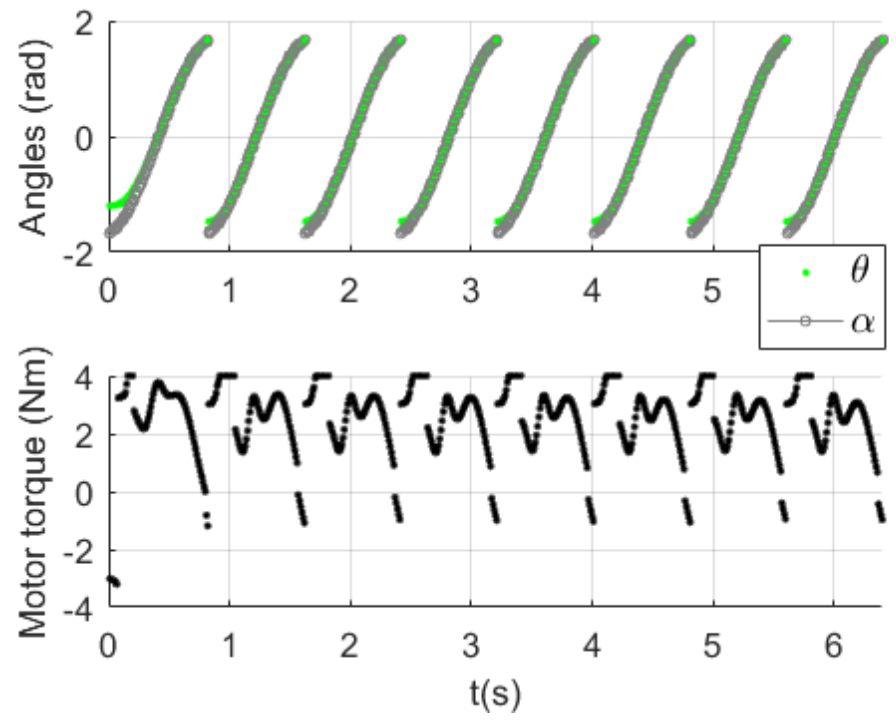

Fig. 5. Numerical experiment 7: The top panel shows the synchronization of $\theta(t)$ and $\alpha(t)$ in eight successful upward brachiating strides with the sliding-mode controller. The bottom panel shows the elbow joint's motor torque with torque capability limited by the sliding-mode boundary, about $[-4,4] \mathrm{Nm}$. 


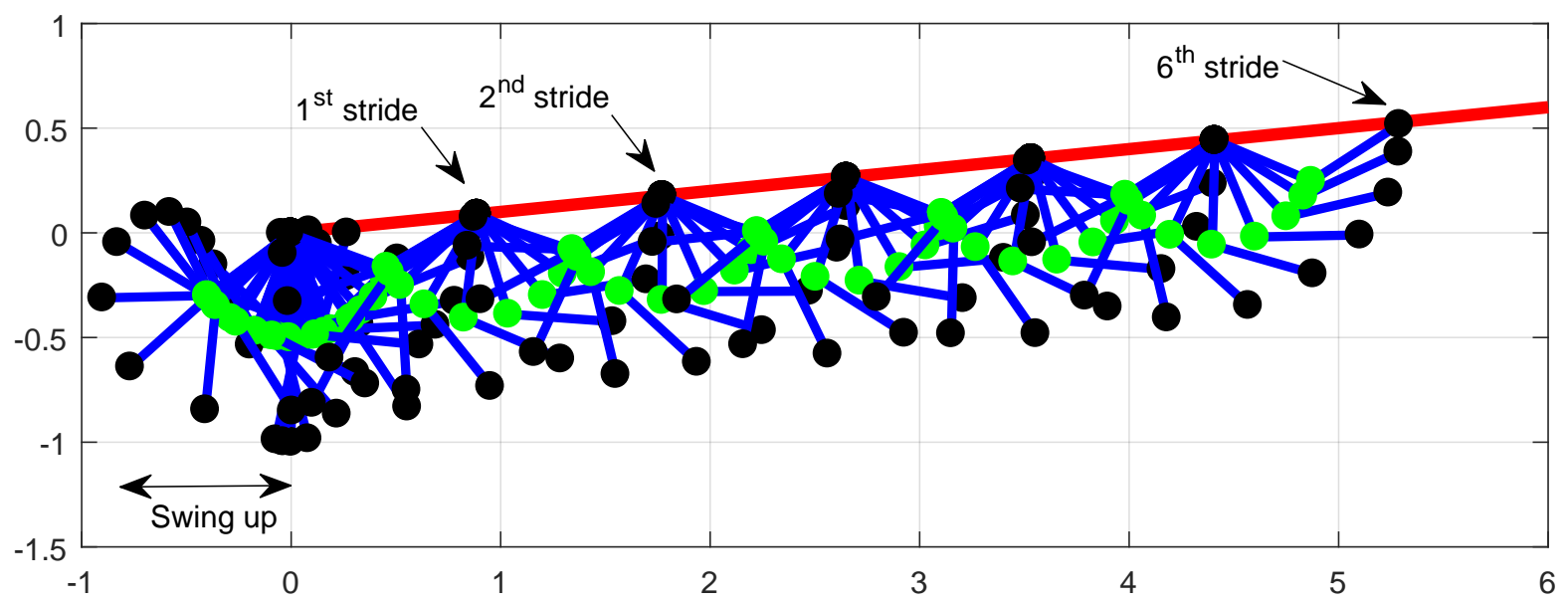

Fig. 6. Numerical experiment 6: Configurations of the brachiator during the six upward brachiating strides with the feedback-linearization scheme.

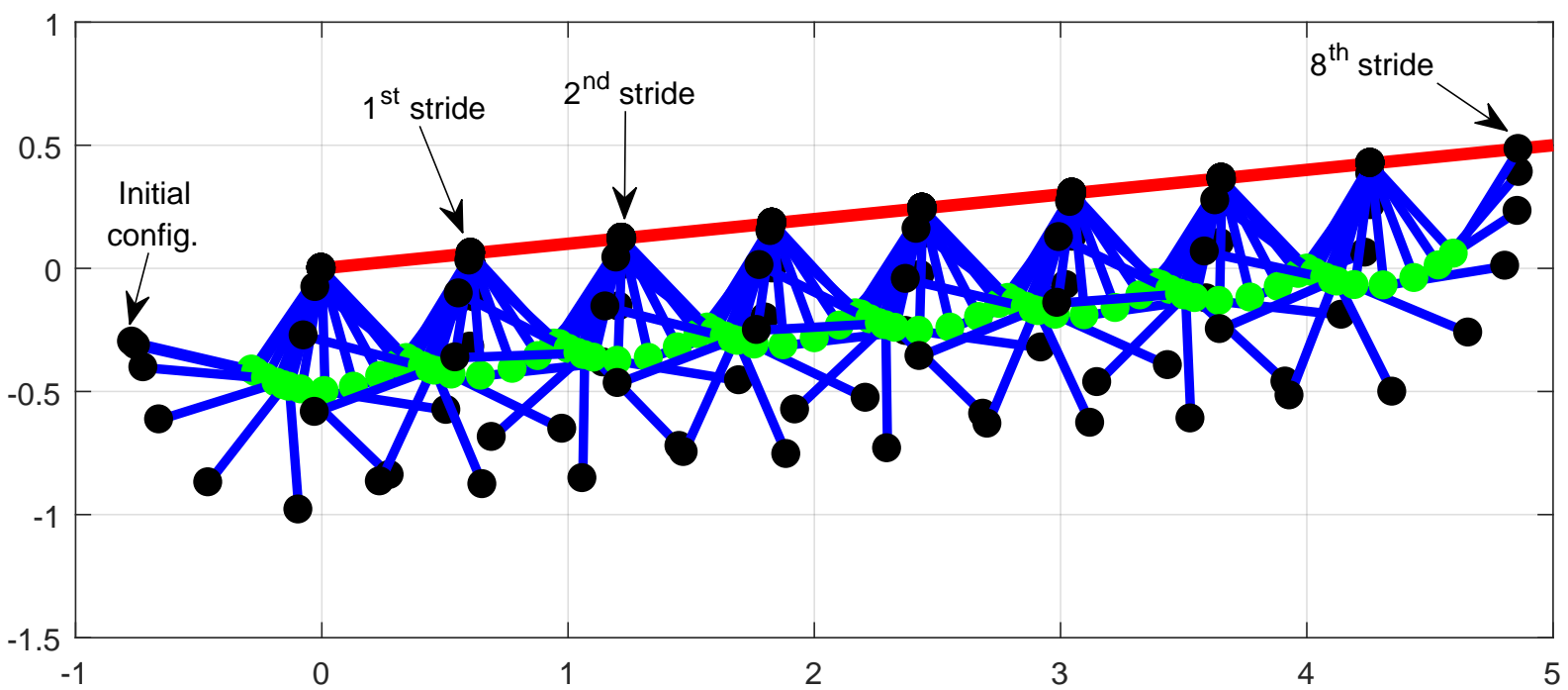

Fig. 7. Numerical experiment 7: Configurations of the brachiator during the eight upward brachiating strides with the sliding-mode controller.

[6] M. W. Gomes and A. L. Ruina, "A five-link 2d brachiating ape model with life-like zero-energy-cost motions," Journal of Theoretical Biology, vol. 237, no. 3, pp. 265-278, 2005.

[7] N. Rosa, A. Barber, R. D. Gregg, and K. M. Lynch, "Stable open-loop brachiation on a vertical wall," in the Proceedings of the IEEE Int'l Conference on Robotics and Automation, pp. 1193-1199, 2012.

[8] N. Rosa and K. Lynch, "The passive dynamics of walking and brachiating robots: Results on the topology and stability of passive gaits," in Nature-Inspired Mobile Robotics: Proceedings of the 16th Int'l Conference on Climbing and Walking Robots and the Support Technologies for Mobile Machines, pp. 633-640, 2013.

[9] N. Rosa and K. M. Lynch, "Extending equilibria to periodic orbits for walkers using continuation methods," in the Proceedings of the IEEE/RSJ Int'l Conference on Intelligent Robots and Systems, pp. 3661-3667, 2014.

[10] Y. Hasegawa, Y. Ito, and T. Fukuda, "Behavior coordination and its modification on brachiation-type mobile robot," in the Proceedings of the IEEE Int'l Conference on Robotics and Automation, vol. 4, pp. 3983-3988, 2000.

[11] T. Fukuda and Y. Hasegawa, "Mechanism and control of mechatronic system with higher degrees of freedom," Annual Reviews in Control, vol. 28, no. 2, pp. 137-155, 2004.

[12] H. Kajima, Y. Hasegawa, T. Fukuda, et al., "Energy-based swingback control for continuous brachiation of a multilocomotion robot," International Journal of Intelligent Systems, vol. 21, no. 9, pp. 10251043, 2006.
[13] T. Fukuda, K. Sekiyama, T. Aoyama, Y. Hasegawa, and Q. Huang, "Locomotion mode transition control of a multi-locomotion robot," A Treatise on Good Robots: Praxiology, Volume 21, vol. 21, p. 83, 2013.

[14] J. Nakanishi, T. Fukuda, and D. E. Koditschek, "Experimental implementation of a" target dynamics" controller on a two-link brachiating robot," in the Proceedings of the IEEE Int'l Conference on Robotics and Automation, pp. 787-792, 1998.

[15] J. Nakanishi, T. Fukuda, and D. E. Koditschek, "A brachiating robot controller," IEEE Transactions on Robotics and Automation, vol. 16, no. 2, pp. 109-123, 2000.

[16] J. Nakanishi, T. Fukuda, and D. E. Koditschek, "Brachiation on a ladder with irregular intervals," Advanced Robotics, vol. 16, no. 2, pp. 147-160, 2002.

[17] M. W. Spong, "The swing up control problem for the acrobot," IEEE Control Systems, vol. 15, no. 1, pp. 49-55, 1995.

[18] V. Utkin, J. Guldner, and J. Shi, Sliding mode control in electromechanical systems, vol. 34. CRC press, 2009.

[19] J.-M. Yang and J.-H. Kim, "Sliding mode control for trajectory tracking of nonholonomic wheeled mobile robots," IEEE Transactions on Robotics and Automation, vol. 15, no. 3, pp. 578-587, 1999.

[20] A. Jusufi, D. I. Goldman, S. Revzen, and R. J. Full, "Active tails enhance arboreal acrobatics in geckos," Proceedings of the National Academy of Sciences, vol. 105, no. 11, pp. 4215-4219, 2008.

[21] T. Libby, T. Y. Moore, E. Chang-Siu, D. Li, D. J. Cohen, A. Jusufi, and R. J. Full, "Tail-assisted pitch control in lizards, robots and dinosaurs," Nature, vol. 481, no. 7380, pp. 181-184, 2012. 